\title{
LIMITS ON THE ENERGY DENSITY OF THE COSMIC INFRARED BACKGROUND
}

\author{
Yoel Rephaeli \\ School of Physics and Astronomy \\ Tel Aviv University \\ Tel Aviv, Israel
}

\author{
Reinhard Schlickeiser \\ Max-Planck Institüt für Radioastronomie \\ Bonn, FRG
}

One of the least known regions of the cosmic electromagnetic background is in the infrared (IR). This infrared background (IRB) is of considerable astrophysical interest. Early stellar activity, either pregalactic (Bond, Carr and Hogan, 1986), or galactic (Partridge and Peebles, 1967), may have contributed significantly to the IRB. More significant, perhaps, are the superimposed IR emissions from normal galaxies, Seyferts, quasars, and rich clusters of galaxies (e.g., Stecker, Puget and Fazio, 1977). There are only few reported measurements of the IRB, which in general yield energy densities comparable to that of the Cosmic Microwave Background (CMB), $\rho_{o} \approx 410^{-13} \mathrm{erg} \mathrm{cm}^{-3}$. An upper limit on the energy density at $2 \mu \mathrm{m}$ is $\delta \equiv \rho / \rho_{o} \leq 1.2$ (Hoffman and Lemke, 1978). Matsumoto, Akiba and Murakami (1984) have detected diffuse emission in the $2-5 \mu \mathrm{m}$ band, with an estimated energy density $\delta=1.2$.

Our poor knowledge of the IRB makes it imperative to use available observational data in order to characterize it. An interesting way to constrain the IRB energy density was suggested by Schlickeiser and Harwit (1982, hereafter SH): Sufficiently intense IRB would Compton scatter relativistic electrons, giving rise to hard $X$ - and $\gamma$-ray emission. SH have used $\gamma$-ray observations of M87 to set limits on the IRB energy density. In a similar analysis, which we briefly summarize here, Rephaeli and Schlickeiser (1988, hereafter RS) have improved these bounds by using recently analyzed HEAO-1 hard X-ray (HXR) measurements of A2319 cluster of galaxies.

CMB and IRB photons can transform to hard X or $\gamma$-rays through Compton scattering off $\mathrm{GeV}$ electrons, whose presence in the IC space of some clusters of galaxies is deduced from radio measurements (see a recent review by Rephaeli, 1988). Since IC soft X-ray emission is predominantly thermal, evidence for the scattering process should be looked for mainly at HXR energies. HEAO-1 A4 data of six Abell clusters have been analyzed (Rephaeli, Gruber and Rothschild, 1987, hereafter RGR; Rephaeli and Gruber, 1988). There is strong evidence for diffuse IC radio emission from these clusters, so HXR emission should be detected at some flux level. But due to insufficient sensitivity of the A4 detectors, we have only upper limits on this emission.

To obtain bounds on the IRB using the HEAO-1 results, one starts with the ejection of electrons to the IC space with an energy distribution characterized by a power-law index $p$. The steady state IC density per unit $\gamma$ (Loretz factor) interval is then $n(\gamma)=n_{0} \gamma^{-(p+1)}$, where $n_{0}$ is related to the rate of ejection of the electrons out of their (galactic) sources. The synchronoton, $F_{s}$, and Compton, $F_{c}$, fluxes are

$$
\begin{gathered}
F_{s}(v)=a_{2}(p) n_{o} R^{3}\left(3 d^{2}\right)^{-1} B^{(p+2) / 2} v^{-(p / 2)}, \\
F_{c}(\varepsilon)=a_{2}(p) a_{3}(p) n_{o} R^{3}\left(3 d^{2)^{-1}}(k T)^{(p+6) / 2} \varepsilon^{-(p / 2)}\right.
\end{gathered}
$$

where $v$ and $\varepsilon$ are the frequency and energy of the radio and HXR photons, $R$ and $d$ are the radius and distance of the source, and $T$ is the effective temperature of the radiation field. If 
the observed radio flux is $F_{s}(v)=A v^{-(p / 2)}$, then the HXR flux is $F_{c}(\varepsilon)$ $=A a_{3}(p)(k T)^{(p+6) / 2} B^{-(p+2) / 2} \varepsilon^{-(p / 2)}$, independent of $R$ and $d$. Knowledge of two out of the three quantities $F_{c}, B$ and $T$ yields the third. If the dominant radiation field is the CMB, for which $T_{o} \approx 2.7 \mathrm{~K}$, then a mean value for $B$ can be obtained from HXR data. The A4 limit on $F_{c}$ thus corresponds to a lower limit on $B$, which we denote by $B_{o}$. We use these formulae to set limits on an IRB which, for simplicity, is represented as a diluted Planckian with energy density $\rho$.

A particularly simple expression for the energy density of the IR field is $\rho / \rho_{0} \leq\left(T_{0} / T\right)^{(p-2) / 2}\left(B / B_{0}\right)^{(P+2) / 2}$. B appears in this equation because the value of the mean IC magnetic field may differ from the lower limit $B_{0}$. A Planckian IR field can be characterized by its peak wavelength $\lambda_{m}$, for which we take the illustrative values $1,10,100 \mu \mathrm{m}$. In A2319, $p=2.8$, and the RGR analysis yields a $2 \sigma$ upper limit is $F_{c} \leq 6.610^{-4} \mathrm{~cm}^{-2} \mathrm{~s}^{-1}$, so that $B_{0} \approx 1.110^{-7}$. A mean IC value of $210^{-7} \mathrm{G}$, was independently determined by Vallee, Broten and MacLeod (1987) from polarization measurements. To get a strict upper limit on IRB energy density, we take $B=210^{-7} \mathrm{G}$ and calculate

$$
\delta \approx\left\{\begin{array}{ll}
0.2 & \lambda_{m=1} 1 \mu \mathrm{m} \\
0.6 & \lambda_{m}=10 \mu \mathrm{m} \\
1.4 & \lambda_{m=100 \mu \mathrm{m}}
\end{array}\right\}
$$

If $B=B_{0}$, the limits are lower by a factor of $(1.8)^{2.4} \approx 4$.

The calculations can be generalized to other clusters for which the mean IC value of $B$ is known. We obtain similar limits using available data on the Coma cluster (RS). These limits should be regarded as conservative bounds on the IRB energy density since clusters have higher density of all conceivable sources of IR radiation. Our limits 10 and $1 \mu \mathrm{m}$ significantly improve on those of SH by factors of about 7 and 50, respectively. At $1 \mu \mathrm{m}$, the above limit is tighter than the observational limit of Hoffman and Lemke (1978), and marginally consistent with the detection by Matsumoto, Akiba and Murakami (1984) of the IRB at $2-5 \mu \mathrm{m}$. From IRAS data, Rowan-Robinson (1986) found that there is an isotropic $100 \mu \mathrm{m}$ component of the IR background, which he identified as extragalactic, with an energy density $\varepsilon \approx 0.17$ at $100 \mu \mathrm{m}$.

Finally, note that when the IRB will be measured, it will then by possible to use our above consideration to determine mean values of magnetic fields in clusters of galaxies.

\section{REFERENCES}

Bond, J.R.. Carr. B.J., and Hogan, C.J. 1986, Astrophys. J., 306, 428.

Hoffman, W., and Lemke, D. 1978, Astron. Astrophys., 68, 389.

Matsumoto, T., Akiba, M., and Murakami, H. 1984, in Advances in Space Research, ed. G.F. Bignami and R.A. Sunyaev (Oxford: Pergamon), 1.

Partridge, R.B., and Peebles, P.J.E. 1967, Astrophys. J., 148, 377.

Rephaeli, Y. 1988, Comments on Astrophys., 12, 265.

Rephaeli, Y., and Schlickeiser, R. 1988, Astron. Astrophys., 194, 99.

Rephaeli, Y., Gruber, D.E., and Rothschild, R.E. 1987, Astrophys. J., 320, 139.

Rephaeli, Y., Gruber, D.E. 1988, Astrophys. J., 333, 133.

Rowan-Robinson, M. 1986, 219, 737.

Schlickeiser, R. and Harwit, M. 1982, Astron. Astrophys., 107, 186.

Stecker, F.W., Puget, J.L., and Fazio, G.G. 1977, Astrophys. J. (Letters), 214, L51.

Vallee, J.P., Broten, N.W., and MacLeod, J.M. 1987, Astrophys. Lett., 25, 181. 